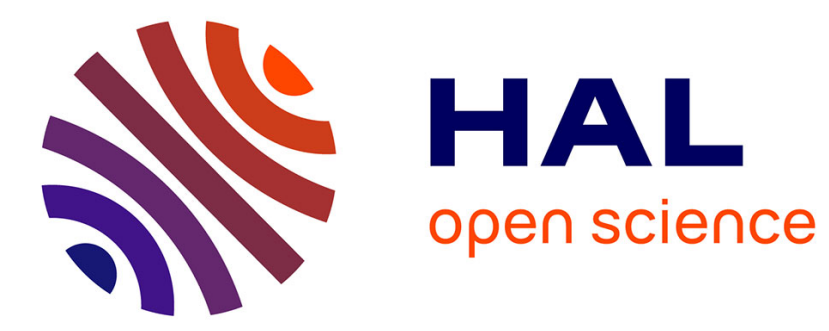

\title{
Nano-laminography for three-dimensional high-resolution imaging of flat specimens
}

L. Helfen, F. Xu, H. Suhonen, L. Urbanelli, P. Cloetens, T. Baumbach

\section{To cite this version:}

L. Helfen, F. Xu, H. Suhonen, L. Urbanelli, P. Cloetens, et al.. Nano-laminography for threedimensional high-resolution imaging of flat specimens. Journal of Instrumentation, 2013, 8, 10 p. 10.1088/1748-0221/8/05/C05006 . hal-01573941

\section{HAL Id: hal-01573941 \\ https://hal.science/hal-01573941}

Submitted on 11 Aug 2017

HAL is a multi-disciplinary open access archive for the deposit and dissemination of scientific research documents, whether they are published or not. The documents may come from teaching and research institutions in France or abroad, or from public or private research centers.
L'archive ouverte pluridisciplinaire HAL, est destinée au dépôt et à la diffusion de documents scientifiques de niveau recherche, publiés ou non, émanant des établissements d'enseignement et de recherche français ou étrangers, des laboratoires publics ou privés. 


\title{
Nano-laminography for three-dimensional high-resolution imaging of flat specimens
}

\author{
L. Helfen, ${ }^{a, b, 1}$ F. Xu, ${ }^{a}$ H. Suhonen, ${ }^{b}$ L. Urbanelli, ${ }^{c}$ P. Cloetens ${ }^{b}$ and T. Baumbach ${ }^{a}$ \\ ${ }^{a}$ ANKA Light Source / Institute for Photon Science and Synchrotron Radiation, \\ Karlsruhe Institute of Technology, \\ PO Box 3640, D-76021 Karlsruhe, Germany \\ ${ }^{b}$ European Synchrotron Radiation Facility (ESRF), \\ BP220, F-38043 Grenoble cedex, France \\ ${ }^{c}$ Dipartimento di Medicina Sperimentale e Scienze Biochimiche, Università di Perugia, \\ 06123 Perugia, Italy
}

E-mail: lukas.helfen@kit.edu

ABSTRACT: Developed for non-destructive three-dimensional (3D) imaging of flat specimens, synchrotron-radiation computed laminography (SR-CL) complements the established method of computed tomography (CT) which is optimised towards rod-like specimens elongated along one direction. SR-CL is realised by the inclination of the tomographic axis with respect to the incident x-ray beam by a defined angle. Its potential for 3D imaging of regions of interests in flat specimens has been demonstrated in various fields of investigation, e.g. in nondestructive device testing, cultural heritage studies, materials science and life sciences. Here we report on the latest developments of SR-CL at the ESRF beamline ID22NI which is dedicated to 3D nano-scale imaging using phase contrast and $\mathrm{x}$-ray fluorescence. For the life sciences, the attractiveness of nano-laminography stems from the ability to study large heterogeneous samples at relatively low resolution in $2 \mathrm{D}$ or $3 \mathrm{D}$ and, subsequently, to focus on the regions of interest for high resolution 3D imaging.

KEYWORDS: Computerized Tomography (CT) and Computed Radiography (CR); Inspection with X-rays; Multi-modality systems; Detection of defects

\footnotetext{
${ }^{1}$ Corresponding author.
} 


\section{Contents}

1 Introduction 1

2 Experimental method 2

3 Application cases in the life sciences 3

3.1 Propagation-based phase-contrast nano-laminography of human cancer cells 4

3.2 Correlative phase-contrast and fluorescence laminography of rat lung tissue exposed to carbon nanotubes $\quad 4$

4 Summary and conclusions $\quad 5$

\section{Introduction}

Inspired by established "tomosynthesis" techniques [1], synchrotron-radiation computed laminography (SR-CL) was developed [2] to provide three-dimensional (3D) imaging of a region of interest (ROI) inside a flat, laterally extended specimen. Originally, SR-CL was set up for high-resolution non-destructive testing of devices [3,4]. In order to complement established laminography methods that use laboratory sources [5,6], a high spatial resolution of $1 \mu \mathrm{m}$ was the primary aim during the early development. In particular, materials sciences could take advantage from these new possibilities [7, 8]. A rotation axis which is tilted with respect to the transmitted beam is the principle difference towards the more commonly applied method of computed tomography $(\mathrm{CT})$ - where the rotation axis is aligned perpendicular to the beam. For flat specimens, the SR-CL geometry yields a more uniform average $\mathrm{x}$-ray transmission over the different projection directions during the scanning rotation (and hence more reliable projection data over the entire scan).

As in CT imaging, the main benefits of using synchrotron radiation over the classical laboratory $\mathrm{x}$ rays arise from additional contrast modes - apart from attenuation - which can be employed. A prominent example is the use of phase contrast [9-11] where phase modulations of the wavefield transmitted by the sample or resulting interference patterns are used for imaging. The implementation of propagation-based phase-contrast laminography $[12,13]$ allowed new specimen types and hence research fields to be addressed, e.g. in cultural heritage studies [14, 15], in paleontology [16], in engineering [17], or in the materials sciences [18, 19]. More recent developments based on Talbot interferometry [20,21] allow efficient and very sensitive phase-contrast imaging at lower spatial resolutions around $5 \mu \mathrm{m}$, e.g. for examining cultural heritage artefacts [22].

Further contrast modes using, e.g., x-ray diffraction for imaging of crystal defects [23], or $\mathrm{x}$-ray fluorescence [24] and the x-ray absorption fine structure [25] for imaging of elemental distributions and binding states are emerging in laminography. Here we show by selected examples that the high sensitivity of phase-contrast laminography allows imaging of single cells and subcellular 
structures as well as sections of biological tissues. The possibility to combine high spatial resolutions and fluorescence imaging could have a high potential for applications in the life sciences and medical research. What makes laminographic imaging particularly attractive in these research fields is that, like in visible-light microscopy, the lateral position of the region of interest (ROI) on the flat, laterally extended specimen can be chosen during the experiment.

\section{Experimental method}

The development of synchrotron laminography [26] was inspired by similar scanning geometries found in device testing [6] and in medical imaging — where corresponding methods are usually called "tomosynthesis" [1]. There exist different acquisition geometries, and early approaches simply integrated an image during a synchronised motion of the source and detector in order to blur out specimen structures which are not situated on the so-called focal plane of the imaging set-up. Today's digital x-ray imaging detectors allow the fast reconstruction of a multitude of cross-sectional slices from a set of individual projection images acquired from different directions of the specimen (usually termed "digital tomosynthesis"). First experiments of tomosynthesis using synchrotron radiation were already reported [27-29] employing different contrast modes. The reconstruction method is mainly based on so-called "shift-and-add" (and sometimes also using shrinking) operations [1] to retrieve a cross-sectional plane from the projection images. This corresponds to algorithms performing so-called unfiltered backprojection [30] in tomography and computed laminography [31] imaging. Other acquisition schemes use a divergent (fan- or cone-beam) geometry to acquire the different projection directions in a set of images which are combined in a reconstruction step (e.g. using algebraic reconstruction techniques [5]) to yield cross-sectional or 3D images. Since the beam divergence at synchrotron imaging set-ups is rather small, these acquisition schemes are not applied there.

In order to facilitate high-resolution imaging at synchrotron facilities, a scanning geometry using a stationary source and detector (and a rotated specimen) was chosen, as is usually the case for SR-CT. This renders the image reconstruction more complicated than in digital tomosynthesis (since simple "shift-and-add" operations are not sufficient). The application of filtered backprojection algorithms and an adapted projection filtering step permits one to dampen over-represented low spatial frequencies [31] which are present in reconstructed images of digital tomosynthesis. The ability to achieve $\mu \mathrm{m}$ resolution at synchrotron imaging set-ups was demonstrated in a number of studies including materials science [32, 33] or device engineering [34].

Due to the long distance between source and experimental station, synchrotron CT set-ups usually operate in a parallel-beam geometry [35]. The trade-off between (energy-dependent) detection efficiency, counting time and spatial resolution permits approximately $0.5 \mu \mathrm{m}$ resolution (at pixel sizes around $0.2 \mu \mathrm{m}$ ) to be achieved in practice via indirect $\mathrm{x}$-ray detection schemes. To overcome this limitation, $\mathrm{x}$-ray microscopy has been proposed in a configuration with Fresnel zone plates as condenser and objective lenses [36]. It presently allows full-field laminographic imaging with a spatial resolution of around $0.5 \mu \mathrm{m}$ on biological specimens.

Here, we describe a different approach based on magnified projection microscopy using the spot of a Kirkpatrick-Baez device as a secondary source, see figure 1. This focusing device can presently be used between 17 and $29 \mathrm{keV}$ x-ray energy [37]. Using typically four sets of projection 


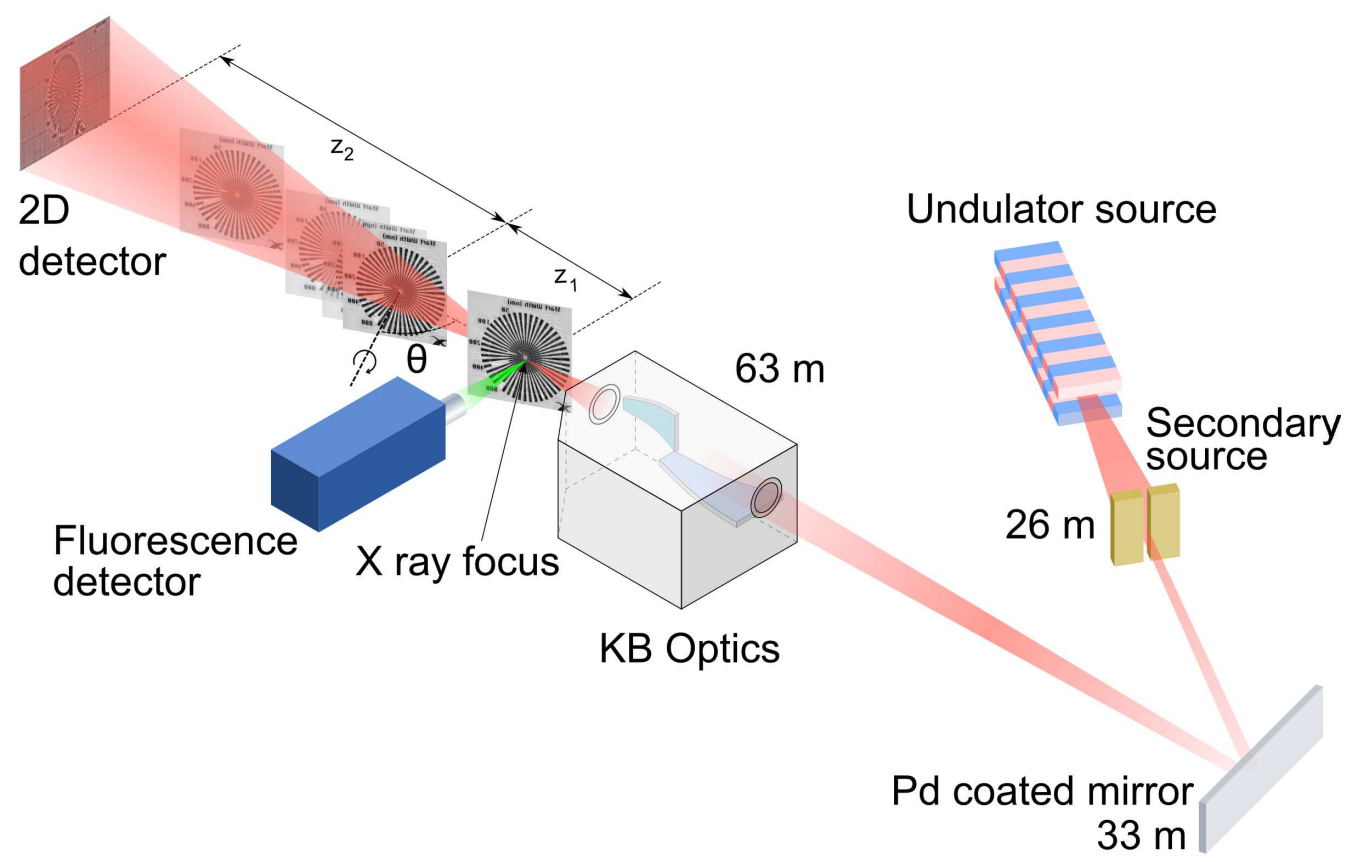

Figure 1. Experimental set-up used for combined x-ray fluorescence and phase-contrast nano-laminography at ESRF end station ID22NI. The specimen can be moved along the optical axis from the focal plane for fluorescence stepping towards the 2D x-ray pixel detector for in-line phase contrast imaging.

radiographs (e.g. approx. 2000 radiographs at each of the four different sample-detector distances), a spatial resolution of around $130 \mathrm{~nm}$ has been achieved [38] in the reconstructed cross-sectional laminography slices (measured in-plane via a lithographically produced Siemens star test pattern). This approach requires phase-retrieval techniques to be applied [39] but it can be combined in a rather straightforward way with fluorescence scanning. For phase imaging, simply translating the specimen along the optical axis permits zooming into the region of interest with adjustable spatial resolution [40]. Fluorescence imaging with variable stepping resolution is performed in the focal plane, allowing in consequence for correlative imaging of structure and composition.

The principal differences compared to synchrotron CT are that for CL the rotation axis is inclined at an angle $\theta<90^{\circ}$ (typically $60^{\circ}$ ) with respect to the optical axis and only a limited ROI is imaged inside the laterally extended specimen. Different plate-like specimen categories have been identified where this data acquisition scheme provides more reliable projection data (and thus more faithful reconstructed cross sections or 3D images) than limited-angle CT [31, 41].

\section{Application cases in the life sciences}

By the following two examples we illustrate that laminographic imaging can be also useful in the life sciences and medical research. The first example demonstrates the ability of nanolaminography to image the spatial distribution of cells inside an epoxy matrix, the second example the potential for imaging of intact tissue sections at selected ROIs in combining morphological and elemental information. 


\subsection{Propagation-based phase-contrast nano-laminography of human cancer cells}

In this example, the ability of laminographic imaging of single cells in a homogeneous matrix is demonstrated. Human cancer cells from the cell line SKOV3 were prepared [42] and embedded in epoxy between two $\mathrm{Si}_{3} \mathrm{~N}_{4}$ membranes (total specimen thickness $80 \mu \mathrm{m}$ ). Four distances between the $\mathrm{x}$-ray focal plane and the tilted specimen rotation axis $\left(\theta=60^{\circ}\right)$ were chosen at an $\mathrm{x}$-ray energy of $17.5 \mathrm{keV}$. At each distance, 1000 magnified projection radiographs (smallest effective pixel size $60 \mathrm{~nm}$ ) were recorded and the phase modulation in the specimen plane retrieved [39, 40] for each projection direction. The result of the laminographic 3D reconstruction (performed by a filtered-backprojection developed in-house [43]) is shown in figure 2. We see that the cells can be easily identified in the matrix (image A) and that they present a subcellular structure, most likely due to the nucleus. The segmented cells and subcellular structure (slice B) are rendered three-dimensionally in image $\mathrm{C}$. A future aim would be to image the 3D subcellular distribution of CdSeZn quantum dots (as has already been demonstrated by 2D fluorescence mapping [42]) which serve as a fluorescence label for specific antibodies against the cancer marker "human epidermal growth factor receptor 2" to perform cancer diagnosis and investigate possible therapeutic routes.

\subsection{Correlative phase-contrast and fluorescence laminography of rat lung tissue exposed to carbon nanotubes}

This example demonstrates the feasibility of local 3D imaging of chemical elements along with the morphology in prepared tissue sections. The aim is to image the excess iron due to exposure of the lung tissue to carbon nanotubes (CNT) and show its spatial distribution inside the tissue. The nanotubes contain iron impurities (10.1 mass-\%) and we detect this excess iron by 3D fluorescence imaging.

For this, single-walled carbon nanotubes (SWCNT) were instilled in rat lungs following a well established animal preparation protocol [44]. A $40 \mu \mathrm{m}$ thick lung tissue specimen embedded in paraffin was first screened (at $17 \mathrm{keV}$ x-ray energy, 1200 projections of $300 \mathrm{~nm}$ pixel size) with a large field of view by low-resolution phase-contrast imaging (figure 3A) and a rather coarse resolution (1.8 $\mu \mathrm{m}$ step size) 2D fluorescence map (figure 3B). Fluorescence hotspots indicate CNT agglomerations in some cellular structures, and we zoomed into one of these areas for laminographic high resolution imaging using $50 \mathrm{~nm}$ pixel size. Two slices at different orientation are chosen from the reconstructed 3D data (see figure 3C) for closer inspection. In the reconstructed slices $\mathrm{D}$ and $\mathrm{E}$, the morphological information from the phase-contrast data (gray values) is combined with the elemental information from the fluorescence data (color mapped). We see that in both slices $\mathrm{D}$ and $\mathrm{E}$ there is an excess of iron inside specific cellular structures. The rounded shape of the cells, their size (about $12 \mu \mathrm{m}$ ) and location (close to the alveolar surface), and the shapes of their nuclei (single, round) can be clearly attributed to alveolar macrophages (AM). The excess content of iron inside some of the AMs indicates the presence of nanotubes. This confirms the finding from electron microscopy that AMs are able to phagocyte nanotubes from the lungs [45]. Further investigations have shown [38] that although most of the nanotubes are cleared by the AMs, some iron is also detected in the alveolar walls and pneumocytes. 

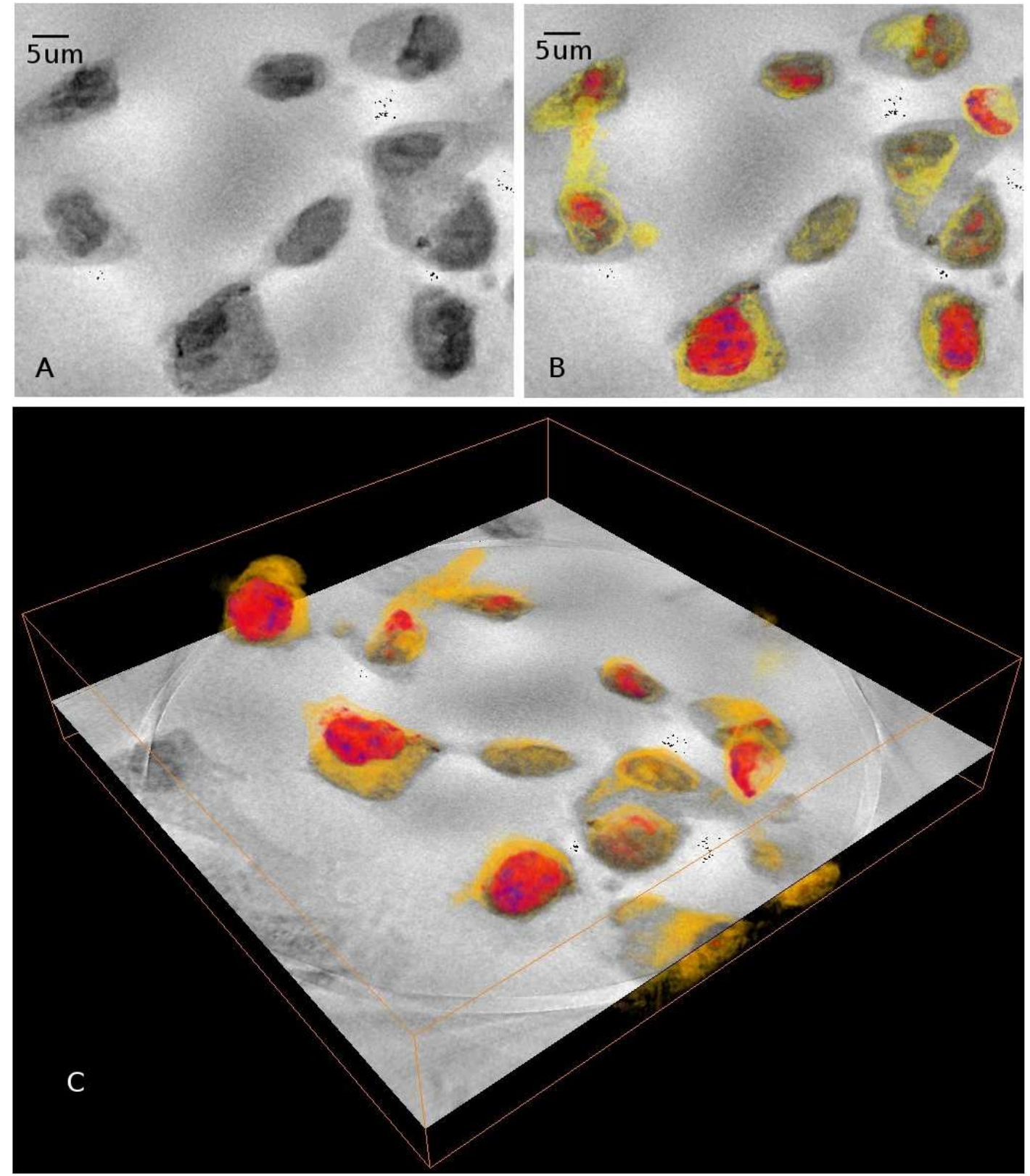

Figure 2. Cross-sectional slices (A) and (B) and 3D rendering (C) by nano-laminography of human SKOV3 cancer cells embedded in epoxy showing a cellular substructure which presumably stem from the cells' nuclei. The size of the 3D rendering (performed with the software Avizo, Visualization Sciences Group SAS, Mérignac, France) is $90 \times 90 \times 21 \mu \mathrm{m}^{3}$ (voxel size $60 \mathrm{~nm}$ ).

\section{Summary and conclusions}

We have implemented nano-laminography at ESRF endstation ID22NI. The present instrumentation allows us to attain a spatial resolution on the scale of $100 \mathrm{~nm}$. The use of reflective KirkpatrickBaez multilayer mirrors as focusing device enables relatively fast scanning times (approx. 2 hours for morphological information via phase-contrast imaging and approx. 12 hours for obtaining 3D 

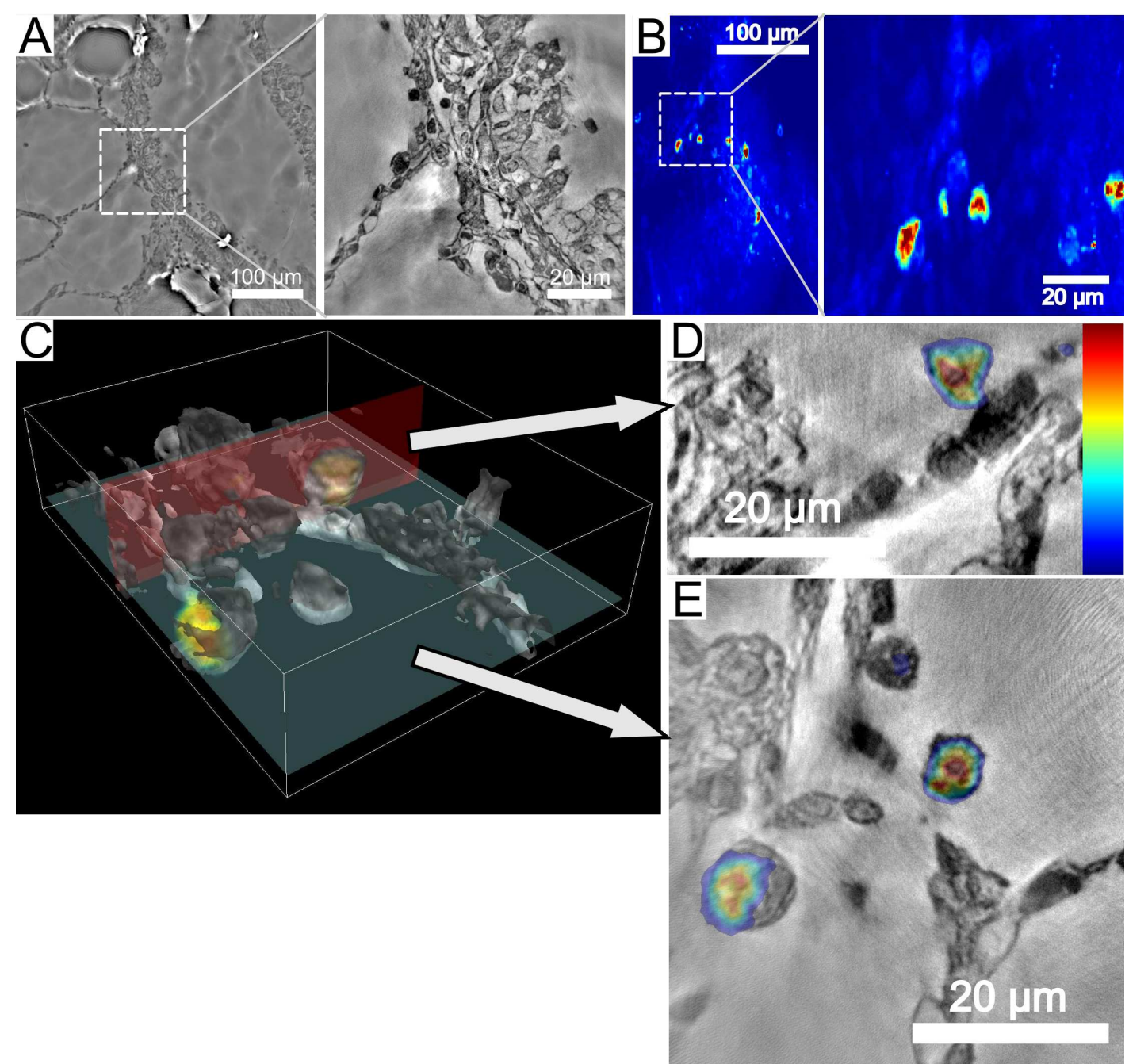

Figure 3. Nano-laminography inspection (x-ray energy $17 \mathrm{keV}$ ) of rat lung tissue embedded in paraffin to detect iron-containing carbon nanotubes. The cross-sectional slice (A) was acquired at $300 \mathrm{~nm}$ voxel size and the zoom-in at $50 \mathrm{~nm}$. The 2D fluorescence maps (B) show iron hot spots under a given projection angle (stepping size $1.8 \mu \mathrm{m}$ ). The reconstruction of the Fe concentration from 64 such viewing angles (stepping size $500 \mathrm{~nm}$ ) is superimposed in the 3D rendering (C) and in the extracted reconstructed slices (D) and (E) with the morphological information from phase-contrast nano-laminography $(50 \mathrm{~nm}$ voxel size).

elemental distributions). Promising applications in life sciences encompass cell imaging where the spatial distribution inside a given volume and their subcellular structure can be obtained.

Furthermore, tissue sections can be investigated by low-resolution scans and individual regions of interest be studied into detail with higher spatial resolutions. In particular, the biodistribution of carbon nanotubes that were instilled in rat lungs could be studied in an intact tissue section by correlative nano-laminography. These abilities could be beneficial in general biotoxicity studies where trace elements and heterogeneities like hot spots of chemical elements could be imaged in three dimensions inside large cell tissue specimens. 


\section{Acknowledgments}

The authors would like to thank the ID22 team for their work in maintaining this highly performant imaging beamline. Dan Elgrabli is acknowledged for preparation of the rat lung specimen. Special thanks go to Wolfgang Ludwig for his precious help in implementing the algebraic reconstruction algorithm, and to Pascal Bernard for the design and integration of the laminography set-up at ID22NI. The authors acknowledge ESRF for providing beamtime in the framework of proposals MI-979 and MI-1029.

\section{References}

[1] J.T. Dobbins III and D.J. Godfrey, Digital x-ray tomosynthesis: current state of the art and clinical potential, Phys. Med. Biol. 482003 R65.

[2] L. Helfen et al., High-resolution three-dimensional imaging of flat objects by synchrotron-radiation computed laminography, Appl. Phys. Lett. 86 (2005) 071915.

[3] L. Helfen et al., Investigation of hybrid pixel detector arrays by synchrotron-radiation imaging, Nucl. Inst. Meth. A 563 (2006) 163.

[4] L. Helfen et al., Synchrotron-radiation computed laminography for high-resolution three-dimensional imaging of flat devices, Phys. Stat. Solidi A 204 (2007) 2760.

[5] J. Zhou, M. Maisl, H. Reiter and W. Arnold, Computed laminography for materials testing, Appl. Phys. Lett. 68 (1996) 3500.

[6] A.R. Kalukin and V. Sankaran, Three-dimensional visualization of multilayered assemblies using x-ray laminography, IEEE Trans. Compon. Pack. Manuf. Technol. 20 (1997) 361.

[7] V. Maurel et al., Three-dimensional investigation of thermal barrier coatings by synchrotron-radiation computed laminography, Scripta Mater. 66 (2012) 471.

[8] D.J. Bull, L. Helfen, I. Sinclair, S. M. Spearing and T. Baumbach, A synthesis of multi-scale $3 d x$-ray tomographic inspection techniques for assessing carbon fibre composite impact damage, Compos. Sci. Technol. 75 (2013) 55.

[9] K.A. Nugent, T.E. Gureyev, D.F. Cookson, D. Paganin and Z. Barnea, Quantitative phase imaging using hard x rays, Phys. Rev. Lett. 77 (1996) 2961.

[10] P. Cloetens et al., Observation of microstructure and damage in materials by phase sensitive radiography and tomography, J. Appl. Phys. 81 (1997) 5878.

[11] D. Paganin, S.C. Mayo, T.E. Gureyev, P.R. Miller and S.W. Wilkins, Simultaneous phase and amplitude extraction from a single defocused image of a homogeneous object, J. Microscopy 206 (2002) 33.

[12] L. Helfen, T. Baumbach, P. Cloetens and J. Baruchel, Phase-contrast and holographic computed laminography, Appl. Phys. Lett. 94 (2009) 104103.

[13] Feng Xu et al., Synchrotron radiation computed laminography for polymer composite failure studies, J. Synchrotron Radiat. 17 (2010) 222.

[14] K. Krug et al., Relics in medieval altarpieces? Combining $x$-ray tomographic, laminographic and phase-contrast imaging to visualize thin organic objects in paintings, J. Synchrotron Radiat. 15 (2008) 55. 
[15] P. Reischig, L. Helfen, A. Wallert, T. Baumbach and J. Dik, High-resolution non-invasive 3d imaging of paint microstructure by synchrotron-based x-ray laminography, Appl. Phys. A 111 (2013) 983.

[16] A. Houssaye, F. Xu, L. Helfen, V. De Buffrénil, T. Baumbach and P. Tafforeau, Three dimensional pelvis and limb anatomy of the Cenomanian hind-limbed snake Eupodophis descouensi (Squamata, Ophidia) revealed by synchrotron-radiation computed laminography,

J. Vertebr. Paleontol. 31 (2011) 2.

[17] V. Maurel et al., Three-dimensional damage evolution measurement in tbc using synchrotron laminography, Oxid. Met. 79 (2013) 313.

[18] L. Helfen et al., Synchrotron and neutron laminography for three-dimensional imaging of devices and flat material specimens, Int. J. Mater. Res. 103 (2012) 170.

[19] Y. Shen et al., Three-dimensional quantitative in situ study of crack initiation and propagation in AA6061 alloy sheets via synchrotron laminography and finite-element simulations, Acta Mater. 61 (2013) 2571.

[20] S. Harasse, N. Hirayama, W. Yashiro and A. Momose, X-ray phase laminography with Talbot interferometer, Proc. SPIE 7804 (2010) 780411.

[21] S. Harasse, W. Yashiro and A. Momose, Iterative reconstruction in x-ray computed laminography from differential phase measurements, Opt. Express 19 (2011) 16560.

[22] V. Altapova et al., Phase contrast laminography based on Talbot interferometry, Opt. Express 20 (2012) 6496.

[23] D. Hänschke, L. Helfen, V. Altapova, A. Danilewsky and T. Baumbach, Three-dimensional imaging of dislocations by x-ray diffraction laminography, Appl. Phys. Lett. 101 (2012) 244103.

[24] N. Watanabe, M. Hoshino, K. Yamamoto, S. Aoki, A. Takeuchi and Y. Suzuki, X-ray fluorescence micro-tomography and laminography using an x-ray scanning microscope,

J. Phys. Conf. Ser. 186 (2009) 012022.

[25] T. Saida et al., 4D visualization of a cathode catalyst layer in a polymer electrolyte fuel cell by $3 D$ laminography-XAFS, Angew. Chem. Int. Ed. 51 (2012) 10311.

[26] L. Helfen, T. Baumbach, P. Pernot, P. Mikulík, M. DiMichiel and J. Baruchel, High-resolution three-dimensional imaging by synchrotron-radiation computed laminography, Proc. SPIE 6318 (2006) 63180N.

[27] D. Shimao, T. Kunisada, H. Sugiyama and M. Ando, Refraction-enhanced tomosynthesis of a finger joint by x-ray dark-field imaging, Jpn. J. Appl. Phys. 46 (2007) L608.

[28] A. Maksimenko, T. Yuasa, M. Ando and E. Hashimoto, Refraction-based tomosynthesis: proof of the concept, Appl. Phys. Lett. 91 (2007) 234108.

[29] D. Shimao, T. Kunisada, H. Sugiyama and M. Ando, Shift-and-add tomosynthesis of a finger joint by $x$-ray dark-field imaging: difference due to tomographic angle, Eur. J. Radiol. 68 (2008) S27.

[30] S. Chilingaryan et al., A GPU-based architecture for real-time data assessment at synchrotron experiments, IEEE Trans. Nucl. Sci. 58 (2011) 1447.

[31] L. Helfen et al., On the implementation of computed laminography using synchrotron radiation, Rev. Sci. Instrum. 82 (2011) 063702.

[32] A.J. Moffat et al., In situ synchrotron computed laminography of damage in carbon fibre-epoxy [90/0](s) laminates, Scripta Mater. 62 (2010) 97. 
[33] T.F. Morgeneyer, L. Helfen, H. Mubarak and F. Hild, 3D digital volume correlation of synchrotron radiation laminography images of ductile crack initiation: An initial feasibility study, Exp. Mech. 53 (2013) 543.

[34] T. Tian et al., Rapid diagnosis of electromigration induced failure time of Pb-free flip chip solder joints by high resolution synchrotron radiation laminography, Appl. Phys. Lett. 99 (2011) 082114.

[35] T. Weitkamp et al., Parallel-beam imaging at the ESRF beamline ID19: current status and plans for the future, AIP Conf. Proc. 1234 (2010) 83.

[36] M. Hoshino, K. Uesugi, A. Takeuchi, Y. Suzuki and N. Yagi, Development of x-ray laminography under an x-ray microscopic condition, Rev. Sci. Instrum. 82 (2011) 073706.

[37] H. Suhonen et al., X-ray phase contrast and fluorescence nanotomography for material studies, Int. J. Mater. Res. 103 (2012) 179.

[38] F. Xu et al., Correlative nanoscale 3d imaging of structure and composition in extended objects, PLOS ONE 7 (2012) e50124.

[39] P. Cloetens et al., Holotomography: Quantitative phase tomography using coherent synchrotron radiation, Appl. Phys. Lett. 75 (1999) 2912.

[40] R. Mokso, P. Cloetens, E. Maire, W. Ludwig and J.-Y. Buffière, Nanoscale zoom tomography with hard $x$ rays using Kirkpatrick-Baez optics, Appl. Phys. Lett. 90 (2007) 144104.

[41] F. Xu, L. Helfen, T. Baumbach and H. Suhonen, Comparison of image quality in computed laminography and tomography, Opt. Express 20 (2012) 794.

[42] S. Corezzi et al., Synchrotron-based X-ray fluorescence imaging of human cells labeled with CdSe quantum dots, Anal. Biochem. 388 (2009) 33.

[43] A. Myagotin, A. Voropaev, L. Helfen, D. Hänschke and T. Baumbach, Fast volume reconstruction for parallel-beam computed laminography by filtered backprojection, manuscript submitted.

[44] D. Elgrabli et al., Effect of BSA on carbon nanotube dispersion for in vivo and in vitro studies, Nanotoxicology 1 (2007) 266.

[45] D. Elgrabli et al., Biodistribution and clearance of instilled carbon nanotubes in rat lung, Part. Fibre Toxicol. 5 (2008) 20. 